\title{
Avaliação do conhecimento de cirurgiões-dentistas sobre hipomineralização molar
}

\section{incisivo}

\author{
Assessment of the knowledge of dentists on incisor molar hypomineralization \\ Evaluación del conocimiento de los odontólogos sobre la hipomineralización de molares incisivos
}

Arlane Maria de Souza Carneiro ORCID: https://orcid.org/0000-0001-7524-7719 Centro Universitário UNIESP, Brasil E-mail: arlanemaria00@gmail.com

Vitor Matheus da Silva Luna

ORCID: https://orcid.org/0000-0002-7490-979X Centro Universitário UNIESP, Brasil E-mail: vitormsl.gba@gmail.com

Naiana Braga da Silva ORCID: https://orcid.org/0000-0001-5976-3561 Centro Universitário UNIESP, Brasil E-mail: naiana.silva@iesp.edu.br Veruska Lima Moura Brasil ORCID: https://orcid.org/0000-0002-0319-0212 Centro Universitário UNIESP, Brasil E-mail: veruskalbrasil@hotmail.com

Lais Guedes Alcoforado de Carvalho ORCID: https://orcid.org/0000-0003-2615-2582 Centro Universitário UNIESP, Brasil E-mail: lais.carvalho@iesp.edu.br

\begin{abstract}
Resumo
Objetivou comparar o perfil de conhecimento sobre HMI entre odontopediatras e clínicos gerais. Este estudo foi do tipo transversal, com aplicação de questionários a 40 cirurgiões- dentistas devidamente registrados no Conselho Regional de Odontologia da Paraíba (CRO-PB), sendo 20 clínicos gerais e 20 especialistas em odontopediatria. A maioria dos participantes dessa pesquisa foi representado por mulheres $(n=36 ; 90,0 \%)$, com idade média de 32,45 anos $( \pm 7,62)$ e tempo de atuação profissional superior a 11 anos. Os dois grupos avaliados obtiveram conhecimento teórico satisfatório em relação à fatores etiológicos, destacando-se o fator genético. Sobre as características clínicas, a maioria respondeu que afeta esmalte e dentina, com opacidades demarcadas, difusa, com localização assimétrica e que afeta alguns dentes. Os dois grupos apresentaram conhecimento teórico, no entanto, a avaliação de imagens mostrou diferença entre os dois grupos. Em relação a conduta clínica, ambos grupos indicam uso de selantes, fluorterapia e restauração em cimento de ionômero de vidro, não havendo difença significativa ( $p>0,05)$. Odontopediatras identificaram corretamente imagens com HMI $(n=13 ; 65,0 \%)$, enquanto os clínicos gerais consideram a mesma imagem como hipoplasia de esmalte $(n=11 ; 55,0 \%)$. Os odontopediatras apresentam maior domínio sobre a HMI em relação ao diagnóstico, porém o conhecimento sobre etiologia, tratamento e escolha de material não apresentou diferença estatisticamente significativa entre os grupos ( $p>0,05)$. Conclui-se que o perfil de conhecimento sobre etiologia, tratamento e características clínicas entre ambos grupos apresentam níveis de conhecimento semelhante. Ao avaliar imagens clínicas, odontopediatras apresentaram padrão de resposta correto, diferentemente de clínicos gerais. Palavras-chave: Odontopediatria; Diagnóstico; Esmalte dentário.
\end{abstract}

\footnotetext{
Abstract

It aimed to compare the profile of knowledge about HMI between pediatric dentists and general practitioners. This was a cross-sectional study, with the application of questionnaires to 40 dentists duly registered with the Regional Council of Dentistry of Paraíba (CRO-PB), being 20 general practitioners and 20 specialists in pediatric dentistry. Most of the participants in this research were women $(n=36 ; 90.0 \%)$, with an average age of 32.45 years $(+7.62)$ and professional experience over 11 years. The two groups evaluated obtained satisfactory theoretical knowledge in relation to etiological factors, especially the genetic factor. Regarding the clinical characteristics, most responded that it affects enamel and dentin, with demarcated opacities, diffuse, with asymmetric location and that affects some teeth. Both groups presented theoretical knowledge, however, the evaluation of images showed a difference between the two groups. Regarding clinical management, both groups indicated the use of sealants, fluortherapy and restoration in glass ionomer cement, with no significant difference ( $p>0.05)$. Pediatric dentists correctly identified images with HMI
} 
$(\mathrm{n}=13 ; 65.0 \%)$, while general practitioners considered the same image as enamel hypoplasia $(\mathrm{n}=11 ; 55.0 \%)$. Pediatric dentists have a greater mastery of HMI in relation to diagnosis, but knowledge about etiology, treatment and choice of material did not show a statistically significant difference between the groups ( $p>0.05)$. It is concluded that the profile of knowledge about etiology, treatment and clinical characteristics between both groups present similar levels of knowledge. When evaluating clinical images, pediatric dentists showed a correct response pattern, unlike general practitioners.

Keywords: Pediatric dentistry; Diagnosis; Dental enamel.

\section{Resumen}

El objetivo fue comparar el perfil de conocimiento sobre HMI entre odontopediatras y médicos generales. Se trata de un estudio transversal, con aplicación de cuestionarios a 40 odontólogos debidamente registrados en el Consejo Regional de Odontología de Paraíba (CRO-PB), siendo 20 médicos generales y 20 especialistas en odontopediatría. La mayoría de los participantes en esta investigación eran mujeres $(n=36 ; 90,0 \%)$, con edad media de 32,45 años $(+7,62)$ y experiencia profesional superior a 11 años. Los dos grupos evaluados obtuvieron conocimientos teóricos satisfactorios en relación a los factores etiológicos, en especial el factor genético. En cuanto a las características clínicas, la mayoría respondió que afecta esmalte y dentina, con opacidades delimitadas, difusas, de localización asimétrica y que afecta a algunos dientes. Ambos grupos presentaron conocimientos teóricos, sin embargo, la evaluación de las imágenes mostró una diferencia entre los dos grupos. En cuanto al manejo clínico, ambos grupos indicaron el uso de selladores, fluoroterapia y restauración en cemento de ionómero de vidrio, sin diferencia significativa ( $\mathrm{p}>0.05)$. Los odontopediatras identificaron correctamente las imágenes con HMI $(\mathrm{n}=13 ; 65,0 \%)$, mientras que los médicos generales consideraron la misma imagen como hipoplasia del esmalte $(\mathrm{n}=11 ; 55,0 \%)$. Los odontopediatras tienen mayor dominio del HMI en relación al diagnóstico, pero el conocimiento sobre etiología, tratamiento y elección del material no mostró diferencia estadísticamente significativa entre los grupos $(\mathrm{p}>0,05)$. Se concluye que el perfil de conocimiento sobre etiología, tratamiento y características clínicas entre los dos grupos presentan niveles de conocimiento similares. Al evaluar las imágenes clínicas, los odontopediatras mostraron un patrón de respuesta correcto, a diferencia de los médicos generales.

Palabras clave: Odontología pediátrica; Diagnóstico; Esmalte dental.

\section{Introduçãa}

A Hipomineralização Molar Incisivo (HMI) trata-se de uma alteração de origem sistêmica, na qual ocorre o comprometimento de forma qualitativa do esmalte dentário de um ou mais primeiros molares permanentes, podendo envolver também os incisivos permanentes igualmente afetados (Fernandes et al., 2012). Sua prevalência a nível mundial varia, amplamente, entre 2,4 a 40,2\% (Jalevik, 2010).

O termo HMI foi estabelecido por Weerhijm, Jalevick e Alalusua em 2001, para especificar a alteração do esmalte do dente provocada por distúrbios que atingem os ameloblastos, durante a fase precoce de maturação amelogênica. $\mathrm{O}$ primeiro relato desta alteração foi na Suécia no final dos anos 70 (Weerhrijm et al., 2001; Jeremias et al., 2010).

O entendimento da HMI permeia a formação dentária, ela surge da interação entre o epitélio oral e o ectomesênquima, por uma sequência de acontecimentos temporários, altamente regulada por um processo denominado Amelogênese. A qual inicia com a formação do esmalte onde os ameloblastos depositam uma matriz orgânica enriquecida com proteína, durante a etapa de secreção. Esta matriz, em seguida, é remodelada pelos estágios de transição e maturação. No decurso de secreção, os ameloblastos na forma de colunas polarizadas adjacentes a formação de esmalte, secretam proteínas como amelogenina e ameloblastos. Esse processo dos secretores na superfície organizam a direção do depósito do esmalte em camadas (Simmer et al., 2010; Simmer et al., 2012; Jeremias et al., 2016).

No decorrer das fases de maturação e mineralização, as proteínas da matriz do esmalte vão sendo degradadas gradativamente através de metaloproteínas-20 da matriz (MMP-20) e calicreína-4 (KLK-4), transformando-se, assim, em prismas de esmaltes simples. No momento em que ameloblastos são sensibilizados no estágio final de mineralização ou maturação da amelogênese, pode ocorrer um defeito na translucidez do esmalte, levando, assim, a hipomineralização de esmalte (Bartlett et al., 2011; Bartlett et al., 2014).

Os ameloblastos são células muitos sensíveis a qualquer alteração sistêmica ou local e que amelogênese pode ser dividida em três estágios, de acordo com a atividade dos ameloblastos: pré- secreção, secreção e maturação. Sendo assim, 
ocorre uma interrupção durante o período de secreção da matriz, propiciando uma redução na espessura do esmalte, tornandose clinicamente um defeito do tipo hipoplasia quantitativa, contudo, se essas células forem afetadas no período de calcificação e/ou maturação, será um defeito qualitativo na translucidez do esmalte, caracterizando uma hipomineralização. Ressalta-se ainda, que há uma suscetibilidade genética podendo está relacionada à patogênese entre variações de genes AMBN, ENAM, TUFT1, TFIP11 e SCUBE1, e a fatores ambientais durante a formação de esmalte, tendo o potencial de interferir na função dos genes, gerando uma predisposição ao HMI (Suckiling 1989; Seow 1997; Barbosa et al., 2017).

A etiologia dessa displasia ainda não foi especificada, contudo estudos executados chegaram a hipóteses de variáveis fatores ambientais, que atuam ao nível sistêmico, incluindo todos os períodos pré-natais, peri natal e durante a infância, podendo intervir com o normal desenvolvimento do esmalte, tendo como exemplos: complicações neonatais, toxinas ambientais, aleitamento materno, desnutrição, doenças respiratórias e frequentes doenças da infância com história de febre alta, sugerindo-os como possíveis fatores etiológicos. Além disso, recentemente tem sido apontado que os fatores genéticos também são uma das principais causas para o desenvolvimento da HMI (Weerheijim et al., 2003; Crombie et al., 2009; Alaluusua 2010).

Clinicamente, a HMI apresenta- se sob a forma de opacidades demarcadas, com bordas claras e distintas do esmalte normal adjacente, e áreas de coloração que vão do branco ao amarelo - acastanhado. De acordo com o grau de severidade, pode apresentar-se como leve, moderada ou severa, com superfície bem demarcada do esmalte sadio, normalmente assimétricos, sendo relatado como "aparência de giz", ou "queijo holandês envelhecido" (Weerheijm et al., 2003).

Este esmalte poroso e frágil é suscetível à fratura sob as forças mastigatórias, resultando ocasionalmente na perda do mesmo, o que pode ocorrer rapidamente após erupção e tornando-se predisposto ao desenvolvimento da lesão cariosa (Weerheijm et al., 2003; Jalevik 2010).

É de suma importância verificar se o molar em erupção apresenta algumas alterações de opacidade ou perda póseruptiva de esmalte, levando a uma supervisão até a erupção por completa de todos os molares, como forma de investigação para redução da perda de esmalte e o risco de presença de cárie (Weerheijm et al., 2013).

Crianças com HMI podem apresentar medo e ansiedade análogo a aqueles sem alteração. Os dentes afetados muitas vezes apresentam hipersensibilidade a estímulos como: escovação, estímulos térmicos e mecânicos, levando a experiência de dor, além de afetar a estética quando há o comprometimento dos incisivos. Orienta-se a aplicação de anestesia local a fim de proporcionar conforto para o paciente, permitindo maior colaboração durante a realização de procedimentos (Mahoney 2001; Jalevik et al., 2002; Weerheijm 2004).

Alguns dos métodos como aplicação de verniz com flúor e selantes ionoméricos poderão contribuir para a redução da sensibilidade. Outrossim, a escovação, educação e conscientização dos pais e da criança são de grande valia, aliados ao tratamento preventivo e interceptativo são de fundamental relevância (Weerheijm 2004; Ruschel et al., 2006).

O objetivo precedente deve ser a redução da dor, considerando o acompanhamento desses dentes longitudinalmente, avaliando tratamentos instituídos e analisando diversos fatores, tais como: a idade do paciente, condições socioeconômicas e grau de severidade do elemento afetado (Oliveira et al., 2015).

Dentre os materiais restauradores, são propostos as resinas compostas e o cimento de ionômero de vidro, podendo resultar a repetidas restaurações, por conta da desintegração do esmalte adjacente ou a presença de outras áreas atingidas. Nesse estágio, a terapia preventiva e restauradora tem sido um grande aliado para prevenir a fratura do esmalte. Visto que, o diagnóstico em períodos posteriores, pode levar a tratamentos mais radicais como endodontias ou exodontias, gerando uma oscilação para casos mais severos (Mahoney 2001; Weerheijm 2004; Ruschel et al., 2006; Oliveira et al., 2015).

Esta alteração apresenta uma enorme relevância clínica, tendo ao longo da última década, captado a atenção dos cirurgiões-dentistas. Devido ao diagnóstico clínico ainda ser muito variável, podendo gerar um equívoco diante a tantas 
alterações de esmalte comuns à coroa dentária, como fluorose e amelogênese imperfeita (Weerheijm 2004; Crombie et al., 2009; Oliveira 2015).

Desse modo, reconhecer as características clínicas da HMI, identificar causas, saber diferenciá-las de outras lesões de esmalte e suceder um diagnóstico precoce são atribuições de grande valia para os cirurgiões- dentistas. Para tal, evidencia-se a dimensão de mais estudos e discussões a cerca desse tema, agregando considerações importantes para o profissional, minimizando o risco de impactos negativos na saúde bucal, além de uma identificação mais fácil, segura e tratamento coerente.

\section{Metodologia}

Trata-se de um estudo transversal, utilizou-se métodos de abordagem indutiva com procedimento estatístico e comparativo (Lakatos \& Marconi, 2009). O universo foi composto por Cirurgiões-dentistas devidamente registrados no Conselhor Regional de Odontologia-Paraíba (CRO-PB) e para compor a amostra, foram selecionados de maneira aleatória 40 cirurgiões-dentistas, divididos igualmente em dois grupos, sendo: G1 $(n=20)$ representado por clínicos gerais e G2 $(n=20)$ representado por Odontopediatras, devidamente registrados no CRO-PB.

Os critérios de inclusão estabelecidos foram: Profissionais que praticam odontologia clínica, devidamente registrado no CRO-PB e atuantes no serviço público e/ou privado. O critério de exclusão estabelecido foi o profissional que negasse participar deste estudo.

Os dados forma coletados através de questionários previamente validado na literatura (Combrie et al., 2008) (Anexo A), enviados pelo Google Forms, em virtude da pandemia pelo COVID-19, para minimizar os riscos de contaminação. Os dados foram coletados no período de maio à julho de 2021.

Este projeto foi submetido e aprovado pelo Comitê de Ética do UNIESP, respeitando todos os princípios da Bioética, segundo o protocolo CAAE: 45618921.7.0000.5184

\section{Resultados}

A variável dependente deste estudo é o conhecimento sobre HMI. Como variável independente, tem-se: especialização odontológica e tempo de atuação como cirurgiã-dentista.

Participaram deste estudo 40 cirurgiões-dentistas, sendo 20 clínicos gerais e 20 odontopediatras, com faixa etária entre 22 e 51 anos ( $M=32,45 ; \mathrm{DP}=7,62)$, majoritariamente do gênero feminino (90\%), tendo a maioria tempo de atuação superior a 11 anos $(27,5 \%)$. A Tabela 1 sumariza as características gerais da amostra. 
Tabela 1 - Caracterização sociodemográfica da amostra por grupos de participantes (clínicos gerais e odontopediatras), n=40, João Pessoa-PB, Brasil, 2021.

\begin{tabular}{cll}
\hline Variáveis & Especialidade & Odontopediatra $(n=20)$ \\
\cline { 2 - 3 } Sexo & Clínico geral $(n=20)$ & $0(0,0 \%)$ \\
Masculino & $4(20,0 \%)$ & $20(100,0 \%)$ \\
Feminino & $16(80,0 \%)$ & $8(40,0 \%)$ \\
Faixa etária & & $7(35,0 \%)$ \\
$<30$ anos & $14(70,0 \%)$ & $4(20,0 \%)$ \\
30 a 40 anos & $5(25,0 \%)$ & $1(5,0 \%)$ \\
41 a 50 anos & $1(5,0 \%)$ & $2(10,0 \%)$ \\
$>50$ anos & $0(0,0 \%)$ & $4(20,0 \%)$ \\
Tempo de atuação & & $5(25,0 \%)$ \\
Até 1 ano & $7(35,0 \%)$ & $9(45,0 \%)$ \\
2 a 5 anos & $6(30,0 \%)$ & $20(100,0 \%)$ \\
6 a 10 anos & $5(25,0 \%)$ & $2(10,0 \%)$ \\
Acima de 11 anos & $20(100,0 \%)$ & \\
\hline
\end{tabular}

Fonte: Autores (2021).

Acerca do conhecimento relacionado à Hipomineralização Molar Incisivo (HMI), todos os profissionais participantes do estudo afirmaram saber o que é a HMI (100\%), tendo a grande maioria dos clínicos gerais aprendido na faculdade (n=14; $70,0 \%)$ e os odontopediatras em cursos ou congressos $(n=14 ; 70,0 \%)$. Além disso, a maioria dos participantes, para ambos grupos, afirmou já ter tido contato com algum caso de HMI na prática clínica (80,0\%), bem como descreveram a HMI como uma alteração frequente na população $(62,5 \%)$. A Tabela 2 apresenta as respostas dos participantes quanto ao conhecimento sobre HMI com base nas especificidades da amostra.

Tabela 2 - Conhecimento relacionado à Hipomineralização Molar Incisivo (HMI) para clínicos gerais e odontopediatras (n=40), João Pessoa-PB, Brasil, 2021.

\begin{tabular}{ccc}
\hline Variáveis & \multicolumn{2}{c}{ Especialidade } \\
\cline { 2 - 3 } Onde aprendeu sobre HMI? & Clínico geral $(n=20)$ & Odontopediatra $(n=20)$ \\
Faculdade & $14(70,0 \%)$ & $4(0,0 \%)$ \\
Cursos/congressos & $2(10,0 \%)$ & $14(70,0 \%)$ \\
Outro & $4(20,0 \%)$ & $10,0 \%)$ \\
Você já teve contato na clínica com algum & & $19(95,0 \%)$ \\
caso de HMI? & $13(65,0 \%)$ & $1(5,0 \%)$ \\
Sim & $7(35,0 \%)$ & $18(90,0 \%)$ \\
Não & & $1(5,0 \%)$ \\
A HMI é uma alteração frequente na & $7(35,0 \%)$ & $1(5,0 \%)$ \\
população? & $12(60,0 \%)$ & $20(100,0 \%)$ \\
Não & $1(5,0 \%)$ & $2(100,0 \%)$ \\
Não soube responder & $20 \%$ & \\
Total & & \\
\hline
\end{tabular}

Fonte: Autores (2021). 
Foi verificado se havia associação entre o tempo de trabalho do cirurgião-dentista e o nível de conhecimento sobre HMI. O teste exato de Fisher demonstrou uma associação estatisticamente significativa entre o tempo de trabalho e conhecimento sobre a frequência de casos de HMI na população $(\mathrm{p}=0,001)$, de modo que quanto maior o tempo de atuação, maior a probabilidade dos profissionais responderem que a HMI é uma alteração frequente na população.

Quando questionados sobre os fatores etiológicos relacionados à HMI, os participantes poderiam selecionar uma ou mais opções, dentre as listadas a seguir: fatores genéticos; medicamentos; condições médicas crônicas; condições médicas agudas; fluoreto; e/ou contaminantes ambientais. Desse modo, a maior parte dos participantes assinalou como opção de resposta mais de uma causa para a etiologia da HMI $(\mathrm{n}=25 ; 62,5 \%)$. Isto é, para a maior parte dos profissionais a etiologia da HMI está relacionada a mais de um fator. A tabela 3 apresenta a categorização de respostas divididas entre os grupos avaliados. As respostas que continham mais de uma opção foram recategorizadas como "mais de um fator".

Tabela 3 - Concepção de clínicos gerais e odontopediatras sobre fatores relacionados à etiologia do HMI para clínicos gerais e odontopediatras, (n=40), João Pessoa-PB, Brasil, 2021.

\begin{tabular}{cccc}
\hline \multirow{2}{*}{ Fatores } & \multicolumn{3}{c}{ Especialidade } \\
\cline { 2 - 4 } & Clínico geral $(n=20)$ & Odontopediatra $(n=20)$ & $P$ \\
\hline Genético & $7(35,0 \%)$ & $3(15,0 \%)$ & $1(5,0 \%)$ \\
Condições médicas crônicas & $0(0,0 \%)$ & $2(10,0 \%)$ & 0,387 \\
Condições médicas agudas & $2(10,0 \%)$ & $14(70,0 \%)$ & $20(100,0 \%)$ \\
Mais de um fator & $11(55,0 \%)$ & $20(100,0 \%)$ & \\
\hline Total & $20 \%$
\end{tabular}

Fonte: Autores (2021).

Nessa direção, foi observada a ausência de associação estatisticamente significativa entre os dois grupos e o conhecimento sobre a etiologia do HMI, em interação com o tempo de serviço profissional $(p=0,387)$. Dessa forma, as respostas dos participantes não estiveram condicionadas à especialidade da profissão, bem como ao tempo de atuação profissional.

Acerca das características clínicas da HMI, os participantes poderiam selecionar uma ou mais opções dentre as listadas a seguir: afeta apenas esmalte; afeta esmalte e dentina; opacidades demarcadas (branco, amarelo, marrom); opacidades difusas; localização simétrica; localização assimétrica; e/ou afeta alguns dentes. De modo geral, a maioria dos profissionais marcou mais de uma opção de resposta ( $\mathrm{n}=32 ; 80 \%)$, destacando-se a opção "afeta esmalte e dentina, opacidades demarcadas, opacidades difusas, localização simétrica, afeta alguns dentes”.

A tabela 4 apresenta a categorização de respostas sobre as características clínicas e dificuldades clínicas relacionadas à HMI para os dois grupos avaliados. As respostas que continham mais de uma opção foram recategorizadas como "mais de uma característica". Com relação às dificuldades relacionadas à HMI enquanto um problema clínico, encontravam-se como possibilidades de respostas as seguintes opções: resolução da sensibilidade dental; maior suscetibilidade a cárie dental; ocorrência de fraturas das opacidades; obtenção do sucesso a longo prazo das restaurações; ansiedade por conta da sensibilidade dental e tratamentos repetidos; e/ou obtenção de uma anestesia eficiente. Os participantes poderiam escolher mais de uma opção de respostas, sendo essa categoria a mais escolhida pela amostra $(n=31 ; 77,5 \%)$. 
Tabela 4 - Características e dificuldades clínicas relacionadas à HMI para clínicos gerais e odontopediatras (n=40), PessoaPB, Brasil, 2021.

\begin{tabular}{|c|c|c|c|}
\hline \multirow[b]{2}{*}{ Características } & \multicolumn{2}{|c|}{ Especialidade } & \multirow[b]{2}{*}{$P$} \\
\hline & $\begin{array}{l}\text { Clínico geral } \\
\quad(n=20)\end{array}$ & Odontopediatra $(n=20)$ & \\
\hline \multicolumn{4}{|l|}{ Características clínicas relacionadas à HMI } \\
\hline Afeta apenas esmalte & $1(5,0 \%)$ & $0(0,0 \%)$ & \\
\hline Opacidades demarcadas & $2(10,0 \%)$ & $2(10,0 \%)$ & \\
\hline Localização assimétrica & $1(5,0 \%)$ & $2(10,0 \%)$ & 1,000 \\
\hline Mais de uma característica & $16(80,0 \%)$ & $16(80,0 \%)$ & \\
\hline \multicolumn{4}{|l|}{ Dificuldades clínicas relacionadas à HMI } \\
\hline Maior suscetibilidade à cárie dental & $4(20,0 \%)$ & $1(5,0 \%)$ & \\
\hline Ocorrência de fraturas das opacidades & $1(5,0 \%)$ & $1(5,0 \%)$ & 0,420 \\
\hline $\begin{array}{c}\text { Obtenção do sucesso a longo prazo das } \\
\text { restaurações }\end{array}$ & $0(0,0 \%)$ & $2(10,0 \%)$ & \\
\hline Mais de uma opção de resposta & $15(75,0 \%)$ & $16(80,0 \%)$ & \\
\hline Total & $20(100,0 \%)$ & $\begin{array}{c}20 \\
(100,0 \%) \\
\end{array}$ & \\
\hline
\end{tabular}

Teste exato de Fisher*. Fonte: Autores (2021).

O teste exato de Fisher demonstrou a ausência de associação estatisticamente significativa entre os grupos de profissionais (clínicos gerais e odontopediatras) e o conhecimento sobre as características clínicas da HMI, em interação com o tempo de serviço profissional HMI ( $\mathrm{p}>0,05)$.

Com relação à pergunta sobre os molares afetados por HMI normalmente terem indicação de selamento ou selante, a maior parte da amostra afirmou que $\operatorname{sim}(n=27 ; 67,5 \%)$. Não foi observada associação entre as variáveis, nem relação de dependência com o fator tempo de serviço dos participantes $(\mathrm{p}=0,313)$. Acerca da indicação de fluorterapia profissional para molares afetados pela HMI, a maioria da amostra assinalou como resposta a opção sim (n=37; 92,5\%). Não foi observada associação entre o conhecimento dos participantes e demais variáveis de interesse $(p=0,231)$.

A respeito dos materiais indicados para a restauração definitiva de dentes com HMI, os participantes, em sua maioria, indicaram como melhor opção de resposta o Cimento Ionômero de Vidro - CVI (n=21; 52,5\%) (Tabela 5). 
Tabela 5 - Comparação entre os grupos em relação ao tipo de conduta clínica para Molares afetados por HMI, (n=40), PessoaPB, Brasil, 2021.

\begin{tabular}{|c|c|c|c|}
\hline \multirow[b]{2}{*}{ Categorias de respostas } & \multicolumn{2}{|c|}{ Especialidade } & \multirow[b]{2}{*}{$P$} \\
\hline & $\begin{array}{l}\text { Clínico geral } \\
(n=20)\end{array}$ & $\begin{array}{l}\text { Odontopediatra } \\
(n=20)\end{array}$ & \\
\hline \multicolumn{4}{|l|}{ Indicação de selamento ou selante } \\
\hline Sim & $11(55,0 \%)$ & $16(80,0 \%)$ & \\
\hline Não & $5(25,0 \%)$ & $2(10,0 \%)$ & \\
\hline Talvez & $4(20,0 \%)$ & $2(10,0 \%)$ & 0,313 \\
\hline \multicolumn{4}{|l|}{ Indicação de fluorterapia } \\
\hline Sim & $17(85,0 \%)$ & $20(100,0 \%)$ & \\
\hline Não & $3(15,0 \%)$ & $0(0,0 \%)$ & 0,231 \\
\hline \multicolumn{4}{|l|}{ Material restaurador indicado } \\
\hline Cimento de ionômero de vidro (CIV) & $9(45,0 \%)$ & $12(60,0 \%)$ & \\
\hline Resina composta fluida & $1(5,0 \%)$ & $0(0,0 \%)$ & \\
\hline Resina composta convencional & $2(10,0 \%)$ & $1(5,0 \%)$ & 0,704 \\
\hline Mais de um material & $8(40,0 \%)$ & $7(35,0 \%)$ & \\
\hline \multicolumn{4}{|l|}{$\begin{array}{l}\text { Fatores que influenciam na escolha de } \\
\text { material restaurador }\end{array}$} \\
\hline Adesão & $1(5,0 \%)$ & $3(15,0 \%)$ & 0,648 \\
\hline Preservação do tecido dental & $3(15,0 \%)$ & $2(10,0 \%)$ & \\
\hline Sensibilidade & $0(0,0 \%)$ & $1(5,0 \%)$ & \\
\hline Mais de um fator & $16(80,0 \%)$ & $14(70,0 \%)$ & \\
\hline Total & $20(100,0 \%)$ & $20(100,0 \%)$ & \\
\hline
\end{tabular}

Teste exato de Fisher*. Fonte: Autores (2021).

Foi observada, ainda, a visão dos profissionais acerca dos que fatores influenciam a escolha dos materiais restauradores acima indicados. Para a grande maioria (75\%), a escolha dos materiais pauta-se em mais de um dos fatores, destacando-se principalmente a adesão (10\%), a preservação do tecido dental (12,5\%) e sensibilidade (5\%). Com relação a associação entre o cnhecimento sobre materiais, a especialidade profissional, o tempo de atuação dos participantes e demais variáveis, não foi observada a dependência estatística entre essas variáveis ( $p>0,05)$ (Tabela 5).

Ademais, foram avaliadas as respostas dos participantes acerca das alterações com base em diferentes imagens. A imagem "A" representava um caso de amelogênese imperfeita, a "B”, amelogênese imperfeita, a "C", fluorose dental e a "D", HMI.

Com relação à imagem $\mathrm{A}$, o grupo de clínicos gerais considerou ser HMI (65,0\%) e os odontopediatras, amelogênese imperfeita (50,0\%), sendo esta a resposta correta. Na imagem B e C, ambos grupos prevaleceu como resposta a amelogênese imperfeita e fluorose dentária, respectivamente, sedo esses os diganósticos corretos. Finalmente, com relação à imagem D, destacou-se diagnósticos para HMI por parte dos odontopediatras (65,0\%), os clínicos gerais diagnosticaram erroneamente, em sua maioria, como hipoplasia de esmalte $(55,0 \%)$. Não foram observadas associações entre as imagens clínicas para nenhuma das hipóteses de diagnóstico entre os dois grupos avaliados ( $p>0,05)$ (Tabela 6). 
Tabela 6- Respostas de clínicos gerais e odontopediatras à imagens clínicas de alterações dentárias, (n=40), Pessoa-PB, Brasil, 2021.

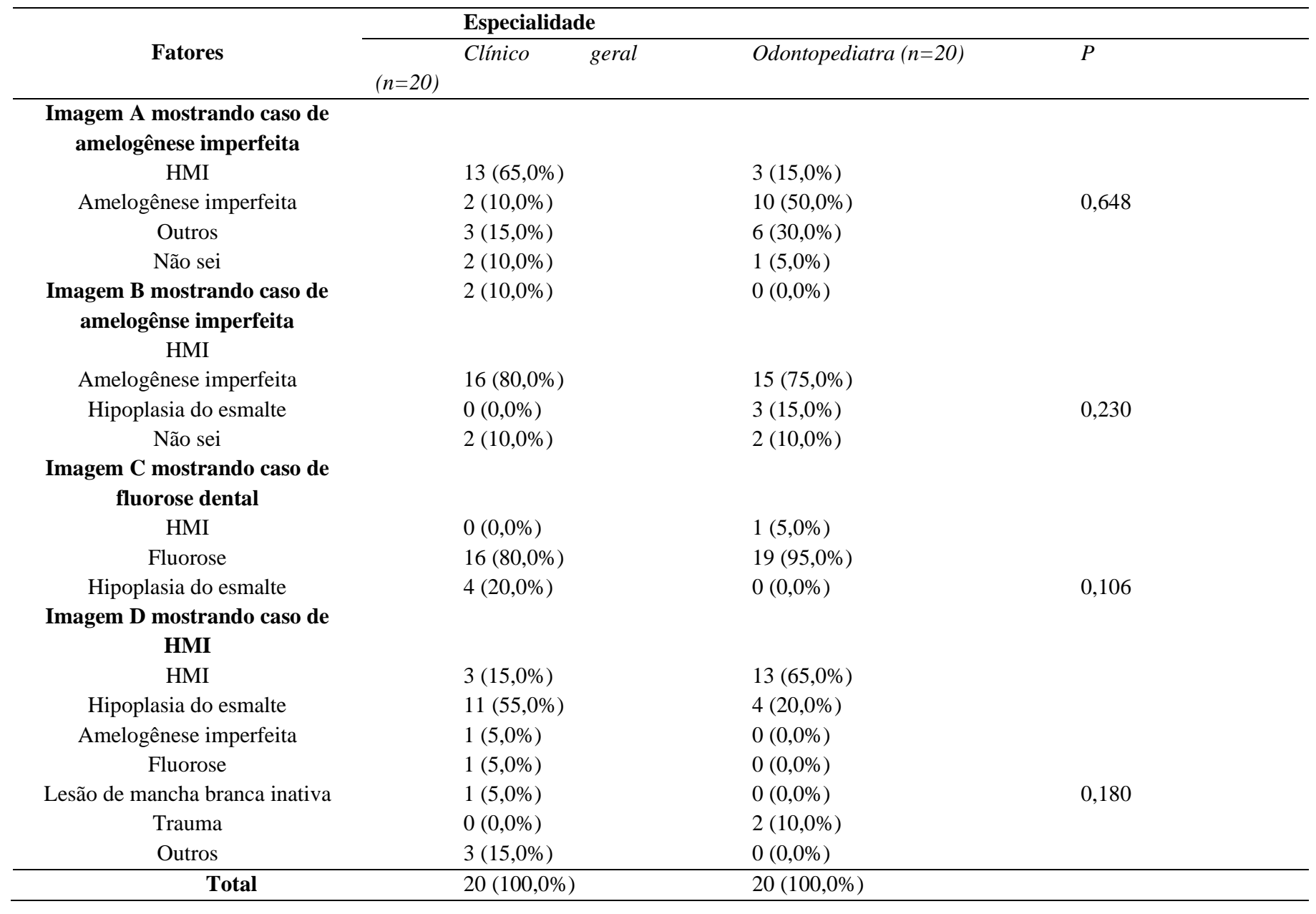

Fonte: Autores (2021).

Foi observada uma associação entre as respostas dadas à patologia representada na Imagem A, a especialidade profissional da amostra e o tempo de atuação dos participantes ( $p=0,003$ ]. Especificamente foi observada uma diferença estatisticamente significativa entre clínicos gerais e odontopediatras quanto as respostas relacionadas a HMI e amelogênese imperfeita, de modo que, na primeira categoria, houve maior endosso dos clínicos gerais, enquanto na segunda (amelogênese imperfeita), houve maior quantitativo de respostas de odontopediatras. Ainda, foi observada a dependência das repostas relacionadas à HMI e o tempo de serviço dos profissionais, sendo observado que os profissionais que possuíam mais de 2 anos de serviço apresentavam maior probabilidade de assinalarem essa opção de respostas $(\mathrm{p}=0,048)$ do que aqueles com histórico de serviço inferior a esse tempo $(\mathrm{p}=0,444)$.

\section{Discussão}

O conhecimento da HMI é extremamente importante na prática clínica, pois é uma condição que acomete, principalmente crianças, com sintomatologia e consequencias à saúde bucal e qualiade de vida. Porém, sabe-se que muitas famílias não tem o hábito de levar crianças ao odontopediatra. Geralmente, a consulta é realizada por algum dentista clínico geral ou de outra especialidade. Diante desse cenário, faz-se importante a realização do presente estudo, a fim de avaliar se existe difenças no perfil de conhecimento entre os dois grupos profissionais, considerando fatores etiológicos, decisão terapêutica e diagnóstico. 
O diagnóstico precoce de dentes com HMI possibilita a adoção de condutas preventivas a fim de evitar desintegração pós-eruptiva do esmalte hipomineralizado, diminuindo a possibilidade de desenvolvimento de lesões cariosas, sensibilidade dentinária, problemas endodônticos, entre outros.

Em consonância aos dados apresentados, observou-se que a maioria dos odontopediatras aprenderam sobre HMI em congressos, tendo em vista que a formação acadêmica destes se deu bem antes a esta condição que vem sendo estudada há relativamente pouco tempo. A HMI apresenta uma abordagem moderadamente atual na odontologia, constituindo um dos defeitos de desenvolvimento de esmalte mais prevalentes ao redor do mundo. De etiologia incerta, torna-se um desafio para o cirurgião dentista devido a sua complexidade de diagnóstico, quando comparada a outras condições semelhantes, e também pelo seu tratamento, que demanda cuidados excepcionais e materiais que se adequem as peculiaridades do esmalte afetado (Araújo et al., 2019).

De acordo com os fatores relacionados à etiologia da HMI os dois grupos avaliados apresentam conhecimento multifatorial da HMI, levando em consideração que todas as opções estavam corretas. Esse resultado mostra que ambos os grupos compreendem a natureza multifatorial, sendo um dado importante do presente estudo.

Destaca-se, contudo, que a etiologia da HMI não está estabelecida na literatura, demonstrando várias suposições, como resultado de variavéis fatores ambientais que influenciam ao nível sistêmico. Inclui-se os presentes nos períodos prénatais, peri natal e durante a infância, que podem interferir no desenvolvimento normal do esmalte, por exemplo: complicações neonatais, toxinas ambientais, aleitamento materno, desnutrição, doenças respiratórians e frequentes doenças de infância, como a febre alta, têm sido sugeridas como possiveis fatores e a predisposição genética (Weerheijim, Mejare 2003; Crombie et al., 2009). Um estudo semelhante foi realizado por Ventura (2019), porém realizando comparação com grupo de alunos. Os resultados também mostraram que a maioria dos estudantes citaram mais de um possível fator etiológico, não havendo difrença entre os grupos avaliados.

Observou-se no referido estudo que a condição genética foi a mais prevalente, considerada como o principal fator etiológico para HMI. O mesmo vai ao encontro dos resultados apresentados em outras literaturas (Ahmadi et al., 2012; Onat, 2013).

Em concordância com Ahmadi et al. (2012) e Onat, (2013), a etiologia da HMI envolve fatores que tem de ser estudados, sob o enfoque de três períodos distintos: período pré-natal, perinatal e neonatal. Tendo em vista, que a condição genética pode ser considerada fator primordial, elencando parto prematuro, desnutrição, nascimento, adentrando ao período de inafância. Entretando faltam evidências na literatura que permitam estabelecer a interação dos fatores etiológicos desta patologia de forma mais clara.

Nessa direção, foi observada a ausência de associação estatisticamente significativa entre os dois grupos e o conhecimento sobre a etiologia do HMI, em interação com o tempo de serviço profissional $(p=0,387)$. Isso significa dizer que as respostas dos participantes não estiveram condicionadas à especialidade da profissão, bem como ao tempo de atuação profissional. Sendo assim, os clínicos gerais participantes do presente estudo, apresentam na formação acadêmica conhecimentos amplos relacionados aos fatores etiológicos. Como justificativa para tal resultado, tem-se a possibilidade de estar associado a currículos reformulados recentemente, tendo em vista que a maioria dos clinicos gerais concluíram a graduação há menos de 5 anos.

Em relação as características clínicas da HMI, os resultados do presente estudo mostraram que 16 participantes (80,0 $\%)$ de ambos grupos (clínicos gerais e odontopediatras) assinalaram mais de uma característica, não havendo diferença significativa entre os dois $(\mathrm{p}>0,05)$. O teste exato de Fisher demostrou a ausência de associação entre esta variável, a especificidade da profissão, bem como o tempo de serviço dos participantes $(\mathrm{p}=0,420)$.

$\mathrm{Na}$ literatura existem dois tipos de estudos realizados similares ao executado, desenvolvendo conhecimento, 
experiência clínica e percepções sobre HMI (Combrie et al.,2008; Cerqueira et al., 2018) tendo como paticipantes cirurgiõesdentistas. Esses estudos mostraram que há falta de conhecimento para o grupo de profissioanais e estudantes. No entanto, esse resultado pode ser justificado pelos estudos não utilizarem um grupo compartativo com odontopediatras, como realizado no presente estudo. Sabe-se que a HMI é uma condição mais frequente na Odontopediatria, sendo rotina em consultórios que apresentam atendimentos direcionadas à crianças, dessa forma, é mais natural que odontopediatras apresentem maior segurança e domínio sobre o assunto.

Outra variável analisada no presente estudo foi a conduta terapêutica e escolha de material restaurador para tratamento de pacientes com HMI. Os resultados aqui apresentados indicaram que o flúor, selante de fóssulas e fissuras e restauração em CIV foram os materiais de escolha para ambos os grupos avaliados. A fluorterapia é uma conduta largamente utilizada em pacientes com HMI. No estudo de William et al., (2006), os autores explanam a magnitude da remineralização e dessensibilização, pois durante a erupção do dente, o esmalte hipomineralizado apresenta grande suscetibilidade a cárie dental e a erosão. Por conseguinte é indicado a remineralização com aplicações de flúor e verniz fluoretado para diminuição da sensibilidade. Além disso, nessa análise é apresentado a importância do uso de selantes em fóssulas e fissuras para a prevenção da cárie dentária e fratura do esmalte pós- erupção, uma das grandes conseuencias da HMI (William et al., 2006; Alves et al., 2021).

A respeito dos materiais indicados para a restauração definitiva de dentes com HMI, os participantes de ambos grupos, em sua maioria, indicaram como melhor opção de resposta o CIV, (52,5\%). A segunda opção mais assinalada foi a predileção de mais de um material. Em conformidade com a literatura, o CIV é considerado um material isolante, liberador de flúor e que favorece a remineralização do tecido. Porém, CIV's convencionais apresentam baixa resistência mecânica, o que impede que seja usado como restaurador definitivo em molares afetados e, por isso, ele tem sido indicado como material provisório em pacientes com pouca maturidade, até que se possa realizar um tratamento mais complexo (Fayle, 2003).

Em uma revisão sistemática que foi pulicada por Sergio et al., (2019) foi possível concluir que não existe, até o momento, nenhuma técnica nem material mais eficaz quando trata-se de HMI. Surege-se que mais estudos sejam realizados para responder a esse questionamento de grande problematização na prática clínica (López-García et al., 2019). Dentre os materiais restauradores o menos indicado é o amálgama devido ao grande desgaste que é feito e a pobre retenção do material nas cavidades atípicas (William et al., 2006).

Em seguida, foi observada a visão os profissionais acerca dos fatores que influenciam a escolha dos materiais restauradores acima indicados. Para a grande maioria (75\%), a escolha dos materiais pauta-se em mais de um dos fatores, destacando-se principalmente adesão, preservação dos tecidos dental e sensibilidade.

A tabela 6 mostra resultados importantes do presente estudo, pois nela, pode ser verificado que, apesar dos CG terem conhecimento teórico sobre etiologia e formas de tratamento, ao avaliar imagens e definir provável diagnóstico, não obtiveram resultados satisfatórios. A imagem "A", refere-se a um caso de amelogênese imperfeita onde a maioria dos ODP acertaram, porém os CG, em sua maioria, acreditaram ser HMI. Na amelogênese imperfeita, os molares são comprometidos da mesma forma nos dentes homólogos, toda a dentição é afetada e há envolvimento genético (Fitzpatrick et al., 2007).

Os dois grupos apresentatam resultados satisfatórios para o diagnóstico de fluorose e amelogênese imperfeita. Provavelmente, por se tratar de duas condições prevalentes na população infantil e adulta. A fluorose dental difere-se da HMI nas opacidades, sendo difusas e afetando toda a dentição, podendo estar diretamente relacionada, na anamnese no ato do preenchimento do prontuário, com o relato do período que houve ingestão em excesso de flúor (Weerheijm et al., 2004; Riatto \& Luna, 2021).

Finalmente, com relação a imagem "D", contendo um caso de HMI, destacou-se diagnósticos para HMI, porém ainda observou-se equívoco com o caso de hipoplasia de esmalte (37,5\%) por parte dos CG. Sendo importante relembrar que, a HMI 
apresenta- se sob a forma de opacidades demarcadas, com bordas claras e distintas do esmalte normal adjacente, e áreas de coloração que vão do branco ao amarelo - acastanhado, com superfície lisa e espessura normal de esmalte. O esmalte hipomineralizado é poroso, sendo frágil e suscetível à fratura quando exposto às forças mastigatórias, e ainda é mais predisposto ao desenvolvimento da lesão cariosa. Esta condição também pode ser observada em segundos molares decíduos (Weerheijm et al., 2003; Jalevik, 2010).

No corrente estudo acerca do conhecimento relacionado à HMI, todos os profissionais participantes do estudo afirmaram ter algum conhecimento sobre tal condição, entretanto observou-se dificuldades com relação ao diagnóstico quando comparou-se o grupo de odontopediatras com clínicos gerais.

De acordo com o estudo de Cerqueira et al., (2018), onde foi analisado o conhecimento dos cirurgiões dentistas sobre a HMI, resultados semelhantes foram encontrados, embora tenham afirmado saber diagnosticar o defeito de esmalte apresentado, observa-se que ainda existe dificuldade para se estabelecer o diagnóstico correto. Isso poderia ser explicado pelo fato de que diagnóstico da HMI é um processo complexo, sendo necessário conhecimento das características clínicas dessas patologias e entendimento da sua classificação, para que outras alterações de esmalte com características semelhantes sejam descartadas.

Dessa forma, destaca-se a importância e o aprofundamento desse tema e suas evidências desde a graduação. No entanto, de acordo com resultados apresentados nesse estudo, observa-se que os clínicos gerais apresentam conhecimento teórico similar aos odontopediatras, porém, nas imagens foram apresentadas divergências no padrão de respostas. Isso reflete que os CG apresentam limitação em diagnóstico clínico, enquanto os ODP possuem maior domínio. Esse resultado pode ser justificado por falta de treinamento e calibre visual em decorrência da falta de aprofundamento científico e prático, uma vez que os ODP teriam como rotina nos seus atendimentos. A HMI exige um diagnóstico diferencial com a fluorose, a amelogênese imperfeita e a hipoplasia, isso justifica algumas respostas com esses diagnósticos.

\section{Conclusão}

Diante dos resultados do presente estudo, é possível considerar que o tempo de trabalho não influenciou no perfil do conhecimento teórico e das características clínicas, acerca da HMI, de clínicos gerais e odontopediatras. Tendo em vista que o grupo de clínicos gerais e odontopediatras conhecem a etiologia da HMI, sendo a etiologia de ordem genética a mais conhecida por ambos os grupos. Não houve diferença estatisticamente significativa entre os dois grupos.

Ambos os grupos conhecem características clínicas associadas à HMI, sendo a opacidade demarcada e localização assimétrica as que mais se destacaram. Em contrapartida, ao avaliarem imagens fotográficas, o grupo de clínicos gerais obtiveram adversidades em realizar diagnóstico, tal fato pode ser explicado pela maior vivência dos odontopediatras em HMI na prática odontológica.

Alem disso, sugere-se a realização de estudos semelhantes, com abordagem nacional e utilizando um maior número amostral, para obter resultados aplicáveis a todo o território nacional e contribuir, dessa forma, no entendimento do perfil do conhecimento sobre HMI entre os dentistas.

\section{Referências}

Ahmadi, R., Ramazani, N., \& Nourinasab, R. (2012). Molar incisor hypomineralization: a study of prevalence and etiology in a group of Iranian children. Iranian journal of pediatrics, 22(2), 245.

Alaluusua, S. (2010). Aetiology of molar-incisor hypomineralisation: a systematic review. European Archives of Paediatric Dentistry, 11(2), 53-58.

de Oliveira Alves, M. C., de Freitas Camargos, B. S., de Amorim Carvalho, T., \& Machado, F. C. (2021). Protocolos clínicos em Hipomineralização Molar Incisivo (HMI): revisão integrativa da literatura. Research, Society and Development, 10(13), e561101321605-e561101321605. 
Araújo, M. V. S. (2019). Hipomineralização molar incisivo: tratamento restaurador e estético.

Bartlett, J. D. \& Simmer, J. P. (2014). Kallikrein-related peptidase-4 (KLK4): papel na formação do esmalte e revelações de camundongos ablacionados. Fronteiras em fisiologia, 5, 240.

Bartlett, J. D., Skobe, Z., Nanci, A., \& Smith, C. E. (2011). Matrix metalloproteinase 20 promotes a smooth enamel surface, a strong dentino-enamel junction, and a decussating enamel rod pattern. European Journal of Oral Sciences, 119, 199-205.

Cerqueira-Silva, R. N., Barbosa-Teixeira, R. J. P., de-Moura, M. S., Branco-Lima, C. C., \& Pereira-Lopes, T. S. (2018). Percepção e condutas de cirurgiõesdentistas sobre a hipomineralização molar-incisivo. CES Odontología, 31(1).

Crombie, F. A., Manton, D. J., Weerheijm, K. L., \& Kilpatrick, N. M. (2008). Molar incisor hypomineralization: a survey of members of the Australian and New Zealand Society of Paediatric Dentistry. Australian dental journal, 53(2), 160-166.

Crombie, F., Manton, D.; Kilpatrick., N. (2009). Etiologia da hipomineralização molar-incisivo: uma revisão crítica. International Journal of Pediatric Dentistry, 19(2), 73-83.

Oliveira, D. C., Favretto, C. O., \& Cunha, R. F. (2015). Molar incisor hypomineralization: Considerations about treatment in a controlled longitudinal case. Journal of Indian Society of Pedodontics and Preventive Dentistry, 33(2), 152.

Fayle, S. A. (2003). Molar incisor hypomineralisation: restorative management. European Journal of Paediatric Dentistry, 4, 121-126.

Fernandes, A. S., Mesquita, P., \& Vinhas, L. (2012). Hipomineralização incisivo-molar: uma revisão da literatura. Revista Portuguesa de Estomatologia, Medicina Dentária e Cirurgia Maxilofacial, 53(4), 258-262.

Fitzpatrick, L., \& O'Connell, A. (2007). First permanent molars with molar incisor hypomineralisation. journal of the Irish Dental Association, 53(1).

Jälevik, B., \& Klingberg, G. A. (2002). Dental treatment, dental fear and behaviour management problems in children with severe enamel hypomineralization of their permanent first molars. International Journal of Paediatric Dentistry, 12(1), 24-32.

Jälevik, B. Prevalence and diagnosis of molar-incisor-hypomineralisation (MIH): a systematic review. European Archives of Paediatric Dentistry, v. 11, n. 2, p. 59-64, 2010.

Jeremias, F., Pierri, R. A., Souza, J. F., Fragelli, C. M. B., Restrepo, M., Finoti, L. S., \& Santos-Pinto, L. (2016). Family-based genetic association for molarincisor hypomineralization. Caries research, 50(3), 310-318.

Jeremias, F., da Costa Silva, C. M., de Souza, J. F., Zuanon, Â. C. C., Cordeiro, R. D. C. L., \& Santos-Pinto, L. (2010). Hipomineralización de incisivos y molares: aspectos clínicos de La severidad. Acta Odontológica Venezolana, 48(4), 23-24.

López-García, S., Pecci-Lloret, M. P., Pecci-Lloret, M. R., Oñate-Sánchez, R. E., García-Bernal, D., Castelo-Baz, P., ... \& Guerrero-Gironés, J. (2019). In vitro evaluation of the biological effects of ACTIVA Kids BioACTIVE restorative, Ionolux, and Riva Light Cure on human dental pulp stem cells. Materials, 12(22), 3694 .

Mahoney, E. K. (2001). Tratamento de defeitos hipoplásicos e hipomineralizados localizados em primeiros molares permanentes. The New Zealand Dental Journal, 97(409), 101-105.

Onat H. \& Tosun G. (2013). Molar incisor hypomineralization. J Pediatr Dent, 1(3), 53-7.

Riatto, S. G., \& Luna, V. M. da S. (2021). Prevalência de fluorose e concentração de fluoreto em água potável. Archives of health investigation, 10(6), 986991. https://doi.org/10.21270/archi.v10i6.5011

Ruschel, H. C., Parizotto, S. P. C. D. O., Amarante, E. C., \& Guedes-Pinto, A. C. (2007). Hipoplasia e hipocalcificação de primeiros molares permanentes. Rev. ABO nac, 89-94.

Seow, W. K. (1997). Diagnóstico clínico de defeitos de esmalte: armadilhas e orientações práticas. International dental journal, 47(3), 173-182.

Simmer, J. P., Papagerakis, P., Smith, C. E., Fisher, D. C., Rountrey, A. N., Zheng, L., \& Hu, J. C. (2010). Regulation of dental enamel shape and hardness. Journal of dental research, 89(10), 1024-1038.

Simmer, J. P., Richardson, A. S., Hu, Y. Y., Smith, C. E., \& Hu, J. C. C. (2012). A post-classical theory of enamel biomineralization... and why we need one. International Journal of Oral Science, 4(3), 129-134.

Suckling, G. W. (1989). Defeitos de desenvolvimento das perspectivas históricas e atuais do esmalte de sua patogênese. Advances in dental research , 3(2), 8794.

Ventura, C. D. O. (2019). Conhecimento dos estudantes de Odontologia sobre a hipomineralização molar incisivo.

Weerheijm, K. L., Jalevik, B., \& Alaluusua, S. (2001). Molar-incisor hypomineralisation. Caries research, 35(5), 390.

Weerheijm, K. L., Duggal, M., Mejàre, I., Papagiannoulis, L., Koch, G., Martens, L. C., \& Hallonsten, A. L. (2003). Judgement criteria for Molar Incisor Hypomincralisation (MIH) in epidemiologic studies: A summary of the European meeting on MIH held in Athens, 2003. European Journal of Paediatric Dentistry, 4, 110-114.

Weerheijm, K. L., \& Mejàre, I. (2003). Molar incisor hypomineralization: a questionnaire inventory of its occurrence in member countries of the European Academy of Paediatric Dentistry (EAPD). International Journal of Paediatric Dentistry, 13(6), 411-416.

Weerheijm, K. L. (2004). Molar incisor hypomineralization (MIH): clinical presentation, aetiology and management. Dental update, 31(1), 9-12.

William, V., Messer, L. B., \& Burrow, M. F. (2006). Molar incisor hypomineralization: review and recommendations for clinical management. Pediatric dentistry, 28(3), 224-232. 